\title{
Molecular Shape Descriptors. 2. Quantitative Structure-Activity Relationships Based Upon Three-Dimensional Molecular Shape Descriptor
}

\author{
Ioan Motoc $^{\text {a,b }}$ and Garland R. Marshall ${ }^{\mathrm{a}}$ \\ Z. Naturforsch. 40a, 1114-1120 (1985); received May 25, 1985 \\ A methodology to incorporate the three-dimensional molecular shape descriptor (3D-MSD) \\ into a quantitative structure-activity relationship is discussed in detail. The 3D-MSD is \\ calculated and correlated with $K_{i \text { app }}$ values for a set of 2,4-diamino-5-benzylpyrimidines which \\ inhibit $E$. coli DHFR. The correlation $(n=22, r=0.95, s=0.214, F=55.10)$ indicates that the \\ polarization interaction dominates the enzyme-inhibitor interactional pattern.
}

\section{Introduction}

The three-dimensional, conformation-dependent molecular shape descriptor $3 \mathrm{D}-\mathrm{MSD}=[\mathrm{OV}, \mathrm{NOV}]$, pairwise related to the overlapping $\left(\mathrm{OV}, \AA^{3}\right)$ and non-overlapping $\left(\mathrm{NOV}, \AA^{3}\right)$ van der Waals volume of molecules, has been reported in a previous paper [1].

In the present paper, we discuss in detail a methodology to generate quantitative structureactivity relationships (QSAR) based upon the 3DMSD and apply the procedure to quantitatively analyse the observed $K_{\text {iapp }}$ values for a set of 5-(substituted-benzyl)-2,4-diaminopyrimidines which inhibit Escherichia coli dihydrofolate reductase (DHFR); the pyrimidine antifolates are important broad-spectrum antibacterial agents [2], antitumor and antimalarial drugs [3]. Consequently, this class of DHFR inhibitors has received the most extensive QSAR study [4], employing a variety of methods: Hansch approach [5-7], Free-Wilson analysis [8], MTD technique [9], distance geometry [10], molecular shape analysis [11], molecular mechanics [12, 13], molecular graphics [14, 15]. This data base may serve, therefore, as a "standard" for evaluating quantitative-structure-activity methods.

\footnotetext{
a Department of Physiology and Biophysics, Washington University School of Medicine, St. Louis, Missouri 63110, USA.

b Department of Computer Science, Washington University, St. Louis, MO 63130, USA.

Reprint request to Dr. Ioan Motoc, Department of Physiology and Biophysics, Box 8101, Washington University School of Medicine, St. Louis, MO 63110, USA.
}

\section{Structure-Activity Correlations Based Upon 3 D-MSD}

The three-dimensional molecular shape descriptor is defined as the twin-number

$$
3 \mathrm{D}-\operatorname{MSD}(\alpha, \beta)=[\mathrm{OV}(\alpha, \beta) ; \operatorname{NOV}(\beta, \alpha)],
$$

where $\operatorname{OV}(\alpha, \beta)$ is the overlapping van der Waals $(\mathrm{VdW})$ volume of the two molecules, and $\operatorname{NOV}(\beta, \alpha)$ is the non-overlapping $\mathrm{VdW}$ volume of molecule $\beta$ superimposed over molecule $\alpha$ according to appropriate criteria. The computer implementation of the $3 \mathrm{D}-\mathrm{MSD}$ approach is based on Eqs. (5), (12), (13) and (14) derived in the previous paper [1]; the program efficiency is greatly improved by using a Simplex optimizer [16] to determine the orientation of the superimposed molecules (the local cartesian framework is standardized) which corresponds to the smallest embedding parallelipiped. Further, the program has been interfaced with the Sybyl software [17]; thereby, obtaining easy access to basic functions as:

(i) Molecular construction: a collection of small molecules (the standard fragment library) provides the building blocks for larger organic molecules; the fragment geometry was obtained from crystallographic data or using standard bond lengther and angles. The molecular model can be visualized and/ or plotted as sticks or space-filling representation.

(ii) Force field calculations, using the White parametrization [18], with a program which allows useful options, e.g., constrain the geometrical relationship among atoms as an aggregate, or constrain the distances between specified atoms [19]. 
(iii) Molecular orbital calculations at semi-empirical (MOPAC package) or ab initio (Gaussian 80) level.

(iv) Comparison of molecules which is performed by overlaying the molecules either as rigid entities (the least-squares fit procedure [20]) or flexible entities (the dynamic fit technique [21]).

\subsection{The reference structure}

Consider the molecules $M_{1}, M_{2}, \ldots, M_{n}$ which exhibit the biological activity via a common mode of action. Let $M_{r}, 1 \leqq r \leqq n$, be the most active compound in the data base considered; then, in agreement with drug-receptor theory [22-24], $M_{r}$ is the best available "copy" of the receptor site features, including the steric ones, and, therefore, it will be considered the reference structure. Consequently, the shape of each molecule $M_{i}, 1 \leqq i \leqq n$, will be characterized by 3D-MSD $(r, i)=[\operatorname{OV}(r, i)$, $\operatorname{NOV}(i, r)]$.

\subsection{The superposition criterion}

Since one is usually operating in the absence of any molecular information concerning the receptor, the basic assumption generally made is a commonality among the molecules considered which explains their bioactivity. The basic tenet is the presence of a receptor which interacts with all molecules under investigation by a similar set of interactions. The three-dimensional arrangement of chemical groups responsible for recognition by the receptor and its subsequent activation has been designated the pharmacophore [25-27]. The pharmacophore concept may be regarded, in the present context, as a general frame of reference for the receptor and, in principle, allows meaningful comparison of congeneric and/or non-congeneric molecules exhibiting the same biological action. Accordingly, our criterion for geometrical congruence of the compared molecule $M_{i}$ over the reference molecule $M_{r}$ imposes that the pharmacophore groups occupy approximately the same position. In the case of conformationally rigid $M_{i}$ 's this is achieved by a straightforward application of Nyburg algorithm [20]. The conformationally flexible molecules impose utilization of a least-squares method combined with molecular mechanics calculations [21]; the way of matching the $n$ molecules includes matching the pharmacophore groups and simultaneous minimization of all molecules, with the coordinates of all atoms being varied. The quality of the geometrical fit and the value of the energy difference between the best fitted conformation and the lowest energy conformation of $M_{i}$ indicate the ability of the molecule to present the pharmacophore in the assumed three-dimensional arrangement - this approach has been used in a study of competitive antagonists of histamine $H_{1}$ receptor [28]). Hence, the $3 \mathrm{D}-\mathrm{MSD}$ is calculated employing the best fitted conformations of $M_{r}$ and $M_{i}$ 's, which, in general, are not energy minima conformers.

\subsection{The three-dimensional molecular shape descriptor}

The receptor and the substrate "feel" the shape and the size of each another through the van der Waals interactions. It follows from the definition of the reference structure that $\mathrm{OV}(r, i)$ represents the "receptor-excluded" volume, i.e., that region of the receptor-active site available for binding by substrate, substrate analogues, or non-substrate analogues, and, therefore, not occupied by the receptor itself. Thus, $\mathrm{OV}(r, i)$ may be regarded as the polarizability volume available to the molecule $M_{i}$; hence, $\mathrm{OV}(r, i)$ is an approximate measure of the (attractive) dispersion interaction between receptor and $M_{i}$. Further, $\operatorname{NOV}(i, r)$ may represent the "receptor-essential" volume, i.e., regions occupied by the receptor and, therefore, not available for binding by other molecules. Hence, we assume that $\operatorname{NOV}(i, r)$ is an approximate averaged measure of the repulsive van der Waals interaction between receptor and $M_{i}$-justification of this assumption is offered by the demonstrated qualitative predictability of the active analog approach [29], the excellent correlations yielded by the steric molecular descriptor [30], steric congestion function [31], cone angle approach [32], or other overlapping methods (for a review see [33]).

Because van der Waals interactions fall off rapidly with distance, the balance between dispersion and repulsion potentials depends to a major degree on the topography of the active site cavity $[34,35]$. Obviously, a molecular shape descriptor of 3D-MSD-type possesses the ability to encompass this feature of the van der Waals interactions. Note that, for example, the molecular shape analysis approach [36], based only on OV $(r, i)$, is in principle 
Table 1. The set of 5 (substituted benzyl)-2,4-diaminopyrimidine inhibitors of E. coli DHFR: the inhibition activity and the values of the $3 \mathrm{D}$-MSD calculated with the compound no. 1 as reference structure.

\begin{tabular}{|c|c|c|c|c|c|}
\hline No. & Compound & $-\log K_{i \text { app }}$ & $\mathrm{OV}(r, i)$ & $\begin{array}{l}\text { 3D-MSD }\left[\AA^{3}\right] \\
\operatorname{NOV}(i, r)\end{array}$ & $\operatorname{NOV}(r, i)$ \\
\hline 1 & $\left.3,4,5-\left(\mathrm{OCH}_{3}\right)_{3}\right)$ & 8.87 & 97.83 & 0.00 & 0.00 \\
\hline 2 & $3-\mathrm{CF}_{3}$ & 7.02 & 49.09 & 15.82 & 48.78 \\
\hline 3 & $3-\mathrm{CH}_{3}$ & 6.70 & 42.63 & 7.85 & 55.17 \\
\hline 4 & 4- $\mathrm{CH}_{3}$ & 6.48 & 46.81 & 3.64 & 51.03 \\
\hline 5 & $3-\mathrm{Cl}$ & 6.65 & 45.23 & 7.27 & 52.61 \\
\hline 6 & $4-\mathrm{Cl}$ & 6.45 & 48.28 & 4.16 & 49.56 \\
\hline 7 & $3-\mathrm{F}$ & 6.23 & 40.52 & 1.99 & 57.31 \\
\hline 8 & $4-\mathrm{F}$ & 6.35 & 41.29 & 1.18 & 56.54 \\
\hline 9 & $\mathrm{H}$ & 6.18 & 36.81 & 1.03 & 61.02 \\
\hline 10 & $4-\mathrm{N}\left(\mathrm{CH}_{3}\right)_{2}$ & 6.78 & 58.52 & 14.07 & 39.35 \\
\hline 11 & $4-\mathrm{NH}_{2}$ & 6.30 & 45.45 & 2.18 & 52.39 \\
\hline 12 & 4-NHCOCH 3 & 6.89 & 52.34 & 24.23 & 45.48 \\
\hline 13 & $4-\mathrm{NO}_{2}$ & 6.20 & 52.67 & 6.39 & 45.16 \\
\hline 14 & $3-\mathrm{OC}_{4} \mathrm{H}_{9}$ & 6.89 & 50.18 & 46.02 & 47.82 \\
\hline 15 & $4-\mathrm{OCF}_{3}$ & 6.57 & 53.62 & 18.72 & 44.24 \\
\hline 16 & $3-\mathrm{OCH}_{2} \mathrm{C}_{6} \mathrm{H}_{5}$ & 6.99 & 55.46 & 60.23 & 42.40 \\
\hline 17 & $3-\mathrm{OCH}_{3}$ & 6.93 & 49.54 & 8.36 & 48.25 \\
\hline 18 & $3,4-\left(\mathrm{OCH}_{3}\right)_{2}$ & 7.72 & 64.52 & 13.28 & 33.32 \\
\hline 19 & $3,5-\left(\mathrm{OCH}_{3}\right)_{2}$ & 8.38 & 67.63 & 10.26 & 30.20 \\
\hline 20 & $4-\mathrm{OCH}_{3}$ & 6.82 & 51.37 & 6.43 & 46.47 \\
\hline 21 & $3-\mathrm{OH}$ & 6.47 & 42.34 & 2.35 & 55.49 \\
\hline 22 & $3,4-(\mathrm{OH})_{2}$ & 6.46 & 48.55 & 3.12 & 49.29 \\
\hline
\end{tabular}

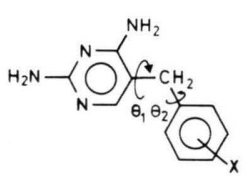

(A)

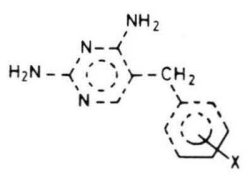

(B)

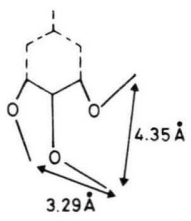

(C)
Fig. 1. The general form of DHFR inhibitors (a); the molecular fragment (solid line) used in 3D-MSD calculations (b); geometrical relations among non-hydrogen atoms of trimethoprime substituents (c).

unable to describe steric effects, while the MTD approach [37], arbitrarily prescribing [38] equal weights * to the attractive and repulsive potentials, lacks the needed flexibility to adequately account for the effect of molecular shape on bioactivity.

* Within the present formalism, MTD is given by [1]: $\operatorname{MTD}=\operatorname{NOV}(r, i)+\operatorname{NOV}(i, r) ;$ but, $\operatorname{OV}(r, i)+\operatorname{NOV}(r, i)$ $=V_{\mathrm{w}, r}$, where $V_{\mathrm{w}, r}$ denotes the van der Waals volume of the reference structure which is constant for the data base under study; accordingly,

$$
\mathrm{MTD}=V_{\mathrm{w}, r}-\mathrm{OV}(r, i)+\operatorname{NOV}(i, r)
$$

The molecular shape descriptor $[\mathrm{OV}(r, i)$, $\mathrm{NOV}(i, r)]$ can be incorporated into a quantitative structure-activity relationship by calculating the regression equation

$Y_{i}=F\left(\sigma_{1 i}, \sigma_{2 i}, \ldots\right)+\zeta \mathrm{OV}(r, i)-\tau \operatorname{NOV}(i, r)$,

where $Y_{i}$ is the estimated bioactivity of $M_{i}, \zeta$ and $\tau$ are the corresponding susceptibilities to the molecular shape features, and $F$ is a function of other molecular features (expressed quantitatively by $\left.\sigma_{1 i}, \sigma_{2 i}, \ldots\right)$ which may also condition the bioactivity.

\section{QSAR Study of DHFR Inhibition by 5-(Substituted-Benzyl)-2,4-Diaminopyrimidines}

The general form of the 22 pyrimidines included in the study (Table 2) is shown in Figure 1 A. Substitutions on the 3 to 5 positions of the benzyl ring were considered, and the observed activities, as measured by $K_{i \text { app }}$ for in vitro inhibition of DHFR from E. coli, span a range of $2.69 \log \left(1 / K_{i \text { app }}\right)$ units.

Trimethoprim, i.e., the molecule no. 1 in Table 1, is the most active inhibitor in the data base and will 
represent the reference structure in calculating the 3 D-MSD values.

The interactions responsible for inhibitor recognition by the DHFR receptor consist in the complex of hydrogen bonds involving the 2,4-diaminopyrimidine moiety, see, e.g. [39, 40]; consequently, this moiety represents the pharmacophore. Hence, the geometrical congruence of the compared inhibitor over trimethoprim will be performed imposing that the 2,4-diaminopyrimidine moiety occupies the same position.

All of the compounds in Table 1 are quite flexible in conformation with respect to $\theta_{1}$ and $\theta_{2}$ (Fig. 1 A), and, as expected, force field calculations show $[11,41]$ that the minimum-energy conformers are close in energy; therefore, it is plausibly to assume that each inhibitor in Table 1 can easily mimic the bioactive conformation of trimethoprim and, accordingly, the conformation with respect to $\theta_{1}$ and $\theta_{2}$ does not represent a critical variable. In order to initiate the 3D-MSD calculations we have frozen the benzyl-2,4-diaminopyrimidine in a minimumenergy conformation characterized by an angle between the two rings of $87.3^{\circ}$. Then, using the dynamical fit technique [21], each inhibitor was superimposed over trimethoprim* and the best fitted conformation used to calculate the 3D-MSD. For all of the compounds in Table 1, the geometrical fit of the benzyl-2,4-diamino-pyrimidine moiety over the same fragment in trimethoprime is practically perfect (i.e., the root mean square values are between 0.098 and $0.327 \AA$ ). The 3D-MSD is calculated, therefore, for the molecular fragment indicated by solid line in Fig. 1B; obviously, the van der Waals volume of the fragment indicated by broken line in Fig. 1 B adds as a constant to $\mathrm{OV}(r, i), 1 \leqq i \leqq n$, hence, it only shifts the origin of $\mathrm{OV}(r, i)$.

The calculations were performed by numerical integration of the van der Waals envelopes [1] using card $\boldsymbol{W}=400,000$ and the effective atomic van der Waals radii given in $[1,42]$. The $3 \mathrm{D}-\operatorname{MSD}(r, i)$ $=[\mathrm{OV}(r, i), \operatorname{NOV}(i, r)]$ and $\operatorname{NOV}(r, i)$ values collected in Table 1 have a standard deviation of about $0.05 \AA^{3}$, as estimated from 22 independent calculations of the van der Waals volume of the reference structure, $V_{\mathrm{w}, r}$, i.e., $V_{\mathrm{w}, r}=\mathrm{OV}(r, i)+\operatorname{NOV}(r, i)$.

* Non-hydrogen substituents were constrained to that shown in Figure $1 \mathrm{C}$.
The 3D-MSD values of Table 1 provide the following equation relating $K_{i \text { app }}$ to the shape characteristics of inhibitor molecules:

$$
\begin{aligned}
\log 1 / K_{i \mathrm{app}}=4.317 & ( \pm 0.067) \\
& (t=64.27) \\
+ & 0.048( \pm 0.005) \mathrm{OV}(r, i) \\
& (t=8.85) \\
+ & 0.003( \pm 0.005) \operatorname{NOV}(i, r) \\
& (t=0.61)
\end{aligned}
$$

with $n=22, r=0.90, s=0.300, F=25.14$. Student $t$ values indicate that only $\mathrm{OV}(r, i)$ is significant, and, consequently, Eq. (1) becomes

$$
\begin{aligned}
\log 1 / K_{\text {iapp }}= & 4.331( \pm 0.066) \\
& (t=65.90) \\
+ & 0.048( \pm 0.005) \mathrm{O}(r, i) \\
& (t=9.11)
\end{aligned}
$$

with $n=22, r=0.90, s=0.301, F=39.40$.

Equation(2) shows, in agreement with experimental evidences [39, 40] and previous QSAR studies [7], that the benzyl substituents point away

Table 2. The values a of 3 D-MSD's $\left[\AA^{3}\right]$ used to derive (3) and (4); the compounds are indexed as in Table 1.

\begin{tabular}{llll}
\hline $\begin{array}{l}\text { Compd. } \\
\text { No. }\end{array}$ & $\mathrm{OV}\left(r_{3}, i\right)$ & $\mathrm{OV}(r, i)$ & $\mathrm{OV}\left(r_{5}, i\right)$ \\
\hline 1 & & & \\
2 & 36.24 & 36.24 & 36.24 \\
3 & 25.88 & 18.02 & 15.93 \\
4 & 20.81 & 16.63 & 15.96 \\
5 & 15.38 & 25.17 & 17.16 \\
6 & 23.32 & 16.61 & 16.09 \\
7 & 15.66 & 26.57 & 17.03 \\
8 & 19.36 & 16.04 & 15.93 \\
9 & 15.67 & 20.42 & 16.10 \\
10 & 15.62 & 16.01 & 15.96 \\
11 & 15.84 & 30.71 & 22.91 \\
12 & 15.51 & 23.58 & 17.29 \\
13 & 15.53 & 26.59 & 21.23 \\
14 & 15.85 & 27.76 & 20.04 \\
15 & 28.91 & 16.01 & 15.96 \\
16 & 16.03 & 32.08 & 16.37 \\
17 & 34.40 & 15.98 & 15.95 \\
18 & 28.24 & 16.14 & 15.90 \\
19 & 30.37 & 28.77 & 16.33 \\
20 & 29.36 & 16.54 & 32.68 \\
21 & 15.85 & 30.19 & 16.28 \\
22 & 21.06 & 16.11 & 15.96 \\
& 20.97 & 21.36 & 17.13 \\
\hline
\end{tabular}

a Note that $\sum_{j=n}^{3} \mathrm{OV}(r, i)>\mathrm{OV}(r, i)$ in Table 1 because $\mathrm{OV}(r, i)$ does not account for the overlapping volume between the benzyl carbon atoms shown in Figure $1 \mathrm{~B}$. 
from the active site and (because the dispersion interaction falls off so rapidly with distance) the atoms beyond the $\mathrm{OCH}_{3}$ group are located too far away from the active site surface to make a contribution to binding. The correlation (2) clearly brings out that the binding via dispersion interaction is fully used by trimethoperim (TMP) and the design of TMP analogues with higher affinity for E. coli DHFR must identify and exploit other enzyme-inhibitor interactions, e.g., as it was attempted in [15].

Inspection of Fig. 1C suggests that the benzyl substituents (most likely the substituent in position 4) may have different contributions to the dispersion interaction due to their possible different location relative to enzyme surface. To test this possibility, the $\mathrm{OV}$ values of the fragments shown in Fig. 1 B have been calculated against the TMP $\mathrm{OCH}_{3}$ group (including the carbon atom adjacent to oxygen) in the 3,4 , and, respectively, 5 positions, and are denoted by $\mathrm{OV}\left(r_{3}, i\right), \mathrm{OV}\left(r_{4}, i\right)$ and, respectively $\mathrm{OV}\left(r_{5}, i\right)$ in Table 2 .

The equation generated by these descriptors is:

$$
\begin{aligned}
& \log 1 / K_{i \mathrm{app}}=3.9620( \pm 0.043) \\
& \\
&(t=81.23) \\
&+ 0.0608( \pm 0.0073) \mathrm{OV}\left(r_{3}, i\right) \\
&(t=8.36) \\
&+ 0.0193( \pm 0.0076) \mathrm{OV}\left(r_{4}, i\right) \\
&(t=2.53) \\
&+ 0.0604( \pm 0.0091) \mathrm{OV}\left(r_{5}, i\right) \\
&(t=6.63)
\end{aligned}
$$

with $n=22, r=0.95, s=0.212, F=39.88$, and the following intercorrelation matrix:

\begin{tabular}{llrll}
\hline & $\log 1 / K_{\text {app }}$ & $\mathrm{OV}\left(r_{3}, i\right)$ & $\mathrm{OV}\left(r_{4}, i\right)$ & $\mathrm{OV}\left(r_{5}, i\right)$ \\
$\log 1 / K_{\text {iapp }}$ & 1.000 & & & \\
$\mathrm{OV}\left(r_{3}, i\right)$ & 0.766 & 1.000 & & \\
$\mathrm{OV}\left(r_{4}, i\right)$ & 0.278 & -0.176 & 1.000 & \\
$\mathrm{OV}\left(r_{5}, i\right)$ & 0.794 & 0.386 & 0.398 & 1.000 \\
\hline
\end{tabular}

Equation (3) and the above matrix indicate an equal contribution to binding of the substituents in the 3 and 5 positions, and a significantly smaller

\begin{tabular}{|c|c|c|c|}
\hline & $\log 1 / K_{i \mathrm{app}}$ & $\begin{array}{l}{\left[\mathrm{OV}\left(r_{3}, i\right)\right.} \\
\left.\quad+\mathrm{OV}\left(r_{5}, i\right)\right]\end{array}$ & $\mathrm{OV}\left(r_{4}, i\right)$ \\
\hline $\begin{array}{l}\log 1 / K_{\text {iapp }} \\
{\left[\mathrm{OV}\left(r_{3}, i\right)\right.}\end{array}$ & $\begin{array}{l}1.000 \\
0.930\end{array}$ & 1.000 & \\
\hline $\begin{array}{l}\left.+\mathrm{OV}\left(r_{5}, i\right)\right] \\
\mathrm{OV}\left(r_{4}, i\right)\end{array}$ & 0.278 & 0.095 & 1.000 \\
\hline
\end{tabular}
contribution of the substituent in position 4 ; lumping together the effect of the former two variables, one obtains the following variant of (3):

$$
\begin{aligned}
& \log 1 / K_{i \mathrm{app}}=3.9650( \pm 0.0479) \\
& \\
&(t=82.42) \\
&+ 0.0604( \pm 0.0047)\left[\mathrm{OV}\left(r_{3}, t\right)\right. \\
&(t=12.69) \\
&+\left.\mathrm{OV}\left(r_{5}, i\right)\right] \\
&+ 0.0200( \pm 0.0075) \mathrm{OV}\left(r_{4}, i\right) \\
&(t=2.66)
\end{aligned}
$$

$n=22, r=0.95, s=0.214, F=55.10$, and the intercorrelation matrix reads as:

Equation (4) represents a clear instance where the systematic consideration of molecular shape leads to a QSAR model in agreement with available experimental evidence [33, 40]. Further, (4) clarifies the meaning of a previous QSAR derived by Hansch et al. [7] using physicochemical parameters; their equation, recomputed for the data base shown in Table 1, reads as:

$$
\begin{aligned}
\log 1 / K_{\text {iapp }}= & 5.692( \pm 0.044) \\
& +1.528( \pm 0.103) \mathrm{MR}_{3,5}^{\prime} \\
& +0.823( \pm 0.140) \mathrm{MR}_{4}^{\prime},
\end{aligned}
$$

$n=22, r=0.96, s=0.194, F=68.21$, with the following intercorrelation matrix:

\begin{tabular}{llrr}
\hline & $\log 1 / K_{i \text { app }}$ & $\mathrm{MR}_{3,5}^{\prime}$ & $\mathrm{MR}_{4}^{\prime}$ \\
$\log 1 / K_{\text {iapp }}$ & 1.000 & & \\
$\mathrm{MR}_{3,5}^{\prime}$ & 0.883 & 1.000 & \\
$\mathrm{MR}_{4}^{\prime}$ & 0.173 & -0.218 & 1.000 \\
\hline
\end{tabular}

$\mathrm{MR}_{3,5}^{\prime}$ stands for the sum of molar refractivity for substituents in the 3 and 5 positions and $\mathrm{MR}_{4}^{\prime}$ is the molar refractivity of substituents in the 4-position only. The prime with MR signifies that the value of MR in each position is limited to the MR value corresponding to $\mathrm{OCH}_{3}$, regardless of the total 
value of MR. The intercorrelation matrix given below clarifies the physical meaning of (5).

\begin{tabular}{lcrrr}
\hline & {$\left[\mathrm{OV}\left(r_{3}, i\right)\right.$} & $\mathrm{OV}\left(r_{4}, i\right)$ & $\mathrm{MR}_{3,5}^{\prime}$ & $\mathrm{MR}_{4}^{\prime}$ \\
{$\left[\mathrm{OV}\left(r_{3}, i\right)\right.$} & $\left.+\mathrm{OV}\left(r_{5}, i\right)\right]$ & & & \\
$\left.+\mathrm{OV}\left(r_{5}, i\right)\right]$ & 1.000 & & & \\
$\mathrm{OV}\left(r_{4}, i\right)$ & 0.095 & 1.000 & & \\
$\mathrm{MR}_{3,5}^{\prime}$ & 0.942 & -0.111 & 1.000 & \\
$\mathrm{MR}_{4}^{\prime \prime}$ & -0.026 & 0.953 & -0.218 & 1.000 \\
\hline
\end{tabular}

A convenient way to further test (4) is to verify how well it predicts the activity of known compounds not included in the data base used to derive the equation. We have done this by using 3,5- $\left(\mathrm{OCH}_{3}\right)_{2}, 4-\mathrm{O}\left(\mathrm{CH}_{2}\right)_{2} \mathrm{OCH}_{3}$ (tetroxoprime, with $\left.\mathrm{OV}\left(r_{3}, i\right)+\mathrm{V}\left(r_{5}, i\right)=57.74, \mathrm{OV}\left(r_{4}, i\right)=25.06\right)$ and 3-I (with $\mathrm{OV}\left(\mathrm{r}_{3}, i\right)+\mathrm{OV}\left(r_{5}, i\right)=44.06, \quad \mathrm{OV}\left(r_{4}, i\right)$ $=23.44)$ for which the estimated $\log 1 / K_{\text {aapp }}$ values are 7.93 and 6.96 , and the observed ones are 8.35 and 7.23 , respectively. Also, the equation quite plausibly suggests that the activity of $3,5-\left(\mathrm{CF}_{3}\right)_{2}$, with $\mathrm{OV}\left(r_{3}, i\right)+\mathrm{OV}\left(r_{5}, i\right)=55.56, \quad \mathrm{OV}\left(r_{4}, i\right)=17.26$,

[1] I. Motoc, G. R. Marshall, R. A. Dammkoehler, and J. Labanowski, Z. Naturforsch. 40 a, 1108 (1985).

[2] B. Roth, in Handbook of Experimental Pharmacology, ed. H. G. Hitching, Springer, Berlin, Vol. 64, Chapter 5, in press.

[3] B. Roth and C. C. Cheng, Prog. Med. Chem. 19, 270 (1982); B. Roth, E. Bliss, and C. R. Beddell, in Molecular Aspects of Anti-Cancer Drug Action, eds. Neidle and Warring, Macmillan, New York 1983.

[4] J. M. Blaney, C. Hansch, C. Silipo, and A. Vittoria, Chem. Rev. 84, 333 (1984).

[5] R. L. Li, C. Hansch, and B. T. Kaufman, J. Med. Chem. 25, 435 (1982).

[6] C. Hansch, R. L. Li, J. M. Blaney, and R. Langridge, J. Med. Chem. 25, 777 (1982).

[7] R. L. Li, S. W. Dietrich, and C. Hansch, J. Med. Chem. 24, 538 (1981).

[8] S. M. Free and J. W. Wilson, J. Med. Chem. 7, 395 (1964).

[9] Z. Simon, I. Badilescu, and T. Racovitan, J. Theor. Biol. 66, 485 (1977).

[10] A. K. Ghose and G. M. Crippen, J. Med. Chem. 27, 901 (1984).

[11] A. J. Hopfinger, J. Med. Chem. 24, 818 (1981).

[12] L. F. Kuyper, B. Roth, D. P. Baccanari, R. Ferone, C. R. Beddell. J. N. Champness, D. K. Stammers, J. C. Dann, F. E. A. Norrington, D. J. Baker, and P. J. Goodford, J. Med. Chem. 25, 1122 (1982).

[13] A. J. Hopfinger, J. Med. Chem. 26, 990 (1983).
3,5- $\mathrm{I}_{2}$, with $\mathrm{OV}\left(r_{3}, i\right)+\mathrm{OV}\left(r_{5}, i\right)=56.74, \mathrm{OV}\left(r_{4}, i\right)$ $=17.66$, or $3,5-\left(\mathrm{OC}_{2} \mathrm{H}_{5}\right)_{2}, 4-\mathrm{OCH}_{3}$, with $\mathrm{OV}\left(r_{3}, i\right)$ $+\mathrm{OV}\left(r_{5}, i\right)=56.11$. OV $\left(r_{4}, i\right)=29.19$, compounds which were not tested in E. coli DHFR system, would compare well with the tetroxoprime activity.

In conclusion, the present paper discusses in detail a methodology to incorporate the three-dimensional molecular shape descriptor 3D-MSD into quantitative structure-activity relationships; it allows for inclusion of structurally diverse and/or conformationally flexible compounds exhibiting common biological action into the conceptual framework of the QSAR approach. The three-dimensional shape descriptors 3D-MSD have been calculated for a set of 2,4-diamino-5-benzylpyrimidines which inhibit E. coli DHFR and led to a significant QSAR. However, the QSAR reported here only partially illustrates the potential of the $3 \mathrm{D}-\mathrm{MSD}$ as the enzyme-inhibitor interactional pattern is dominated by the polarization interaction, the steric effects being inoperative in this case.

\section{Acknowledgement}

This work was supported by NIH grant GM24483.
[14] R. L. Li, C. Hansch, D. Matthews, J. M. Blaney, R. Langridge, T. J. Delcamp, S. S. Susten, and J. H. Freisheim, Quant. Struct. Act. Relat. 1, 1 (1982).

[15] L. F. Kuyper, B. Roth, D. P. Baccanari, R. Ferone, C. R. Beddell, J. N. Champness, D. K. Stammers, J. G. Dann, F. E. A. Norrington, D. J. Baker, and P. J. Goodford, J. Med. Chem. 25, 1120 (1982).

[16] J. A. Nader and R. Mead, Computer J. 7, 308 (1965).

[17] Sybyl Manual, Tripos Assoc., St. Louis, MO, USA.

[18] D. N. J. White, Computers and Chemistry 1, 225 (1977).

[19] J. Labanowski and (i. R. Marshall, in preparation.

[20] S. C. Nyburg, Acta Cryst. B30, 251 (1974).

[21] D. J. Duchamp, in Computer-Assisted Drug Design, eds. E. C. Olson and R. E. Christoffersen, ACS Symp. Ser., Vol. 112, 1979.

[22] C. J. Cavallito, ed., Structure-Activity Relationships, Pergamon, Oxford 1973.

[23] J. M. van Rossum, ed. Kinetics of Drug Action, Springer, Berlin 1977.

[24] W. Bartmann and G. Snatzbe, eds., Structures of Complexes Between Biopolymers and Low Molecular Weight Molecules, Wiley-Heyden, Chichester 1982.

[25] P. Gund, Ann. Rep. Med. Chem. 14, 299 (1979).

[26] C. Humblet and G. R. Marshall, Ann. Rep. Med. Chem. 15, 267 (1980).

[27] G. R. Marshall, in Quantitative Approaches to Drug Design, J. C. Dearden, ed., Elsevier, Amsterdam 1983.

[28] S. Naruto, I. Motoc, and G. R. Marshall, Eur. J. Med. Chem., in press. 
[29] G. R. Marshall, C. D. Barry, H. E. Bosshard, P. A. Dammkoehler, and D. A. Dunn, in Computer-Assisted Drug Design, Symp. Ser. No. 112, American Chemical Society, Washington, D. C. 1979.

[30] I. Motoc, Quant. Struct.-Act. Relat. 3, 43, 47 (1984).

[31] W. T. Wipke and P. Gund, J. Amer. Chem. Soc. 96, 299 (1974): ibid. 98, 8107 (1976).

[32] C. A. Tolman, J. Amer. Chem. Soc. 92, 2956 (1970); C. A. Tolman, W. C. Seidel, and L. W. Gosser, J. Amer. Chem. Soc. 96, 53 (1974).

[33] R. Gallo, Prog. Phys. Org. Chem. 14, 115 (1981).

[34] W. P. Jenks, Proc. Natl. Acad. Sci. USA 78, 4046 (1981).

[35] M. I. Page, in Quantitative Approaches to Drug Design, ed. J. C. Dearden, Elsevier, Amsterdam 1983.
[36] A. J. Hopfinger, J. Amer. Chem. Soc. 102, 7196 (1980).

[37] A. T. Balaban, A. Chiriac, I. Motoc, and Z. Simon, Steric Fit in QSAR, Springer, Berlin 1980.

[38] I. Motoc, Top. Curr. Chem. 114, 93 (1983).

[39] D. A. Matthews, R. A. Alden, J. T. Bolin, S. T. Freer, R. Hamlin, N. Xuong, J. Kraut, M. Poe, M. Williams, and K. Hoogsteen, Science 197, 452 (1977).

[40] J. T. Bolin, D. J. Filman, D. A. Matthews, R. C. Hamlin, and J. Kraut, J. Biol. Chem. 257, 13650 (1982).

[41] I. Motoc, unpublished results.

[42] I. Motoc and G. R. Marshall, Chem. Phys. Lett., in press. 